\title{
A Necessary and Sufficient Condition for Nonnegative Product Linearization of Orthogonal Polynomials
}

\author{
Ryszard Szwarc
}

\begin{abstract}
A necessary and sufficient condition for nonnegative product linearization of an orthogonal polynomial system is derived. The method goes through a discrete hyperbolic boundary value problem associated with the three-term recurrence relation. The paper provides a unified approach to nonnegative linearization problems that comprises results obtained earlier and gives new ones.
\end{abstract}

\section{Introduction}

One of the central problems in the theory of orthogonal polynomials is to decide whether the expansion of the product of two orthogonal polynomials, in terms of these polynomials, has nonnegative coefficients. In other words, we look which orthogonal systems $\left\{p_{n}\right\}$ have the property

$$
p_{n}(x) p_{m}(x)=\sum c(n, m, k) p_{k}(x)
$$

with nonnegative coefficients $c(n, m, k)$ for every choice of $n, m$, and $k$.

This property is a direct generalization of the product formula for trigonometric polynomials

$$
\cos n \theta \cos m \theta=\frac{1}{2} \cos (n-m) \theta+\frac{1}{2} \cos (n+m) \theta .
$$

Certain classical orthogonal polynomials admit explicit computation of the coefficients $c(n, m, k)$. For example, they are known explicitly for the ultraspherical polynomials along with their $q$-analogs [8]. However, they are not available in a simple form for the nonsymmetric Jacobi polynomials (see [7]).

The first general criterion for nonnegativity of linearization coefficients is due to Askey [1]. Although it is pretty strong it is not strong enough to cover all classes of the ultraspherical polynomials. It is well-known that the problem of product linearization is equivalent to a certain discrete hyperbolic boundary value problem. This approach has been used in [6], [9] to derive new criteria for nonnegative linearization. The new criteria

Date received: October 25, 2001. Date revised: August 19, 2002. Date accepted: September 9, 2002. Communicated by Erik Koelink. Online publication: February 21, 2003.

AMS classification: Primary, 42C05, 47B39.

Key words and phrases: Orthogonal polynomials, Recurrence relation, Nonnegative linearization, Discrete boundary value problem. 
are strong enough to cover not only all the ultraspherical polynomials, but also most of the Jacobi polynomials, as well as their $q$-analogues, the associated polynomials, and also many of the Askey-Wilson polynomials.

In this paper we prove that the nonnegative product linearization is equivalent to the existence of a family of special matrices with triangular support. As far as we know, it is the first necessary and sufficient condition for nonnegative linearization. Moreover, this equivalent condition can be easily implemented. Our earlier results [9, Theorem 1] and [6, Theorems 1 and 2] fit into this new approach.

\section{Nonnegative Linearization}

Assume we are dealing with a system of orthogonal polynomials $\left\{p_{n}\right\}_{n=0}^{N-1}$ with respect to a probability measure $\mu$ on the real line. In case the support of $\mu$ is infinite we have $N=+\infty$, otherwise $N$ is the number of points in the support of $\mu$. We assume that the leading coefficient of each $p_{n}$ is strictly positive. Then the polynomials satisfy a three-term recurrence relation of the form

$$
x p_{n}=\gamma_{n} p_{n+1}+\beta_{n} p_{n}+\alpha_{n} p_{n-1},
$$

where $\gamma_{n}, \alpha_{n+1}>0$ and $\beta_{n} \in \mathbf{R}$ for $0 \leq n<N$. We use the convention that $\alpha_{0}=\gamma_{-1}=$ 0 . Moreover, if $N$ is finite we set $\gamma_{N-1}=\alpha_{N}=0$. This form of recurrence relation will be useful for applications. For the proofs of the following results we will need the renormalized polynomials $P_{n}$ defined as

$$
\begin{aligned}
P_{0}(x) & =p_{0}(x), \\
P_{n}(x) & =\frac{\gamma_{0} \gamma_{1} \ldots \gamma_{n-1}}{\alpha_{1} \alpha_{2} \ldots \alpha_{n}} p_{n}(x), \quad 0<n<N .
\end{aligned}
$$

The new polynomials satisfy

$$
x P_{n}=\alpha_{n+1} P_{n+1}+\beta_{n} P_{n}+\gamma_{n-1} P_{n-1}, \quad 0 \leq n<N .
$$

It is clear that the nonnegativity of the product linearization for the systems $\left\{p_{n}\right\}$ and $\left\{P_{n}\right\}$ are equivalent.

Let $u(n, m)$ be a matrix of complex numbers indexed by $0 \leq n, m<N$. Define two operators $L_{1}$ and $L_{2}$ acting on such matrices by the rule

$$
\begin{aligned}
& \left(L_{1} u\right)(n, m)=\alpha_{n+1} u(n+1, m)+\beta_{n} u(n, m)+\gamma_{n-1} u(n-1, m), \\
& \left(L_{2} u\right)(n, m)=\alpha_{m+1} u(n, m+1)+\beta_{m} u(n, m)+\gamma_{m-1} u(n, m-1) .
\end{aligned}
$$

Observe that by the recurrence relation (4), if we take $u(n, m)=P_{n}(x) P_{m}(x)$ for some $x$, then

$$
L_{1} u=x u, \quad L_{2} u=x u .
$$

Thus for such matrices $u$ we have

$$
H u:=\left(L_{1}-L_{2}\right) u=0 .
$$


The last equality also holds for the special matrix

$$
u(n, m)=g(n, m, k)=\left(\int_{\mathbf{R}} P_{k}^{2} d \mu\right)^{-1} \int_{\mathbf{R}} P_{n}(x) P_{m}(x) P_{k}(x) d \mu(x),
$$

where $k$ is fixed. Additionally, we have

$$
u(n, 0)=g(n, 0, k)= \begin{cases}1, & k=n \\ 0, & k \neq n\end{cases}
$$

Hence the matrix $u$ satisfies $H u=0$ and has nonnegative boundary values.

Proposition 1. The polynomials $p_{n}$ admit nonnegative product linearization if and only if every matrix $u=\{u(n, m)\}$ such that

$$
\left\{\begin{array}{l}
H u=0 \\
u(n, 0) \geq 0
\end{array}\right.
$$

satisfies $u(n, m) \geq 0$ for $0 \leq m \leq n<N$.

Proof. The "if" direction is clear, because we can always assume $n \geq m$ and, as we have seen above, the matrix $u(n, m)=g(n, m, k)$ satisfies (6). Assume $P_{n}$ admit nonnegative product linearization, i.e., $g(n, m, k) \geq 0$ (see (5)). Let $u=u(n, m)$ be any solution to (6). Set

$$
\tilde{u}(n, m)=\sum_{k=0}^{N-1} u(k, 0) g(n, m, k)
$$

The series in fact reduces to a finite sum because $g(n, m, k) \neq 0$ only for $|n-m| \leq k \leq$ $\min (n+m, N-1)$. By (5) we have $H \tilde{u}=0$. Moreover,

$$
\tilde{u}(n, 0)=u(n, 0) \text {. }
$$

The boundary values determine the solution uniquely, because the equality $H u=0$ allows us to compute the numbers $u(n, m)$ inductively with respect to $m$. Hence $u(n, m)=$ $\tilde{u}(n, m)$, which implies $u(n, m) \geq 0$.

For each point $(n, m)$ with $1 \leq m \leq n<N$, let $\Delta_{n, m}$ denote the set of lattice points in the plane defined by

$$
\Delta_{n, m}=\{(i, j)|0 \leq j \leq i<N,| n-i \mid<m-j\}
$$


The set $\Delta_{n, m}$ is depicted below for $n+m<N$ (the points in $\Delta_{n, m}$ are marked with open circles).

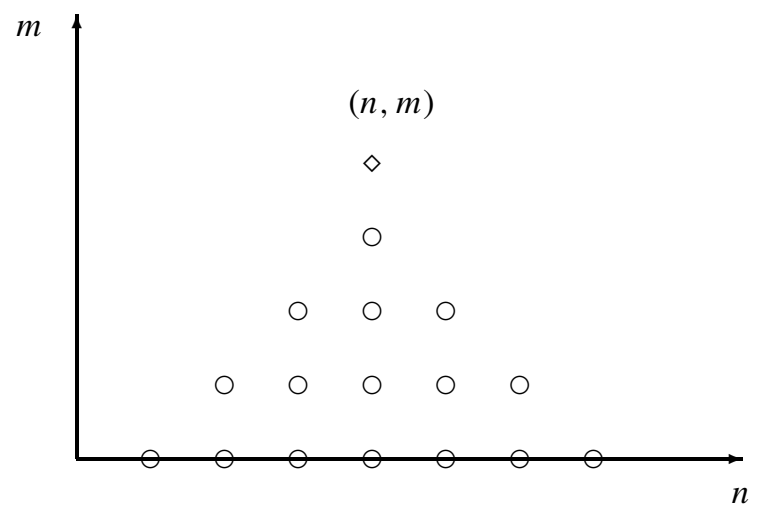

In case $N$ is finite and $n+m \geq N$, the corresponding picture is

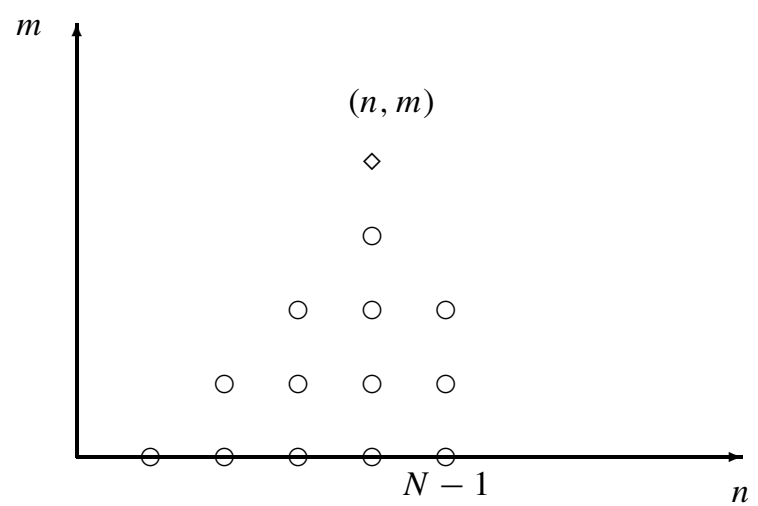

Let $H^{*}$ denote the adjoint operator to $H$ with respect to the inner product of matrices

$$
\langle u, v\rangle=\sum_{n, m=0}^{N-1} u(n, m) \overline{v(n, m)} .
$$

This operator acts according to

$$
\begin{aligned}
\left(H^{*} v\right)(n, m)= & \gamma_{n} v(n+1, m)+\beta_{n} v(n, m)+\alpha_{n} v(n-1, m) \\
& -\gamma_{m} v(n, m+1)-\beta_{m} v(n, m)-\alpha_{m} v(n, m-1) .
\end{aligned}
$$

The main result of this note is the following:

Theorem 1. The orthogonal polynomials $\left\{p_{n}\right\}_{n=0}^{N-1}$, where $N$ may be finite or infinite, admit nonnegative linearization if and only if for every $(n, m)$, with $1 \leq m \leq n<N$, 
there exists a matrix $v_{n, m}(i, j)$ such that:

(i) $\operatorname{supp} v_{n, m} \subset \Delta_{n, m}$.

(ii) $\left(H^{*} v_{n, m}\right)(n, m)<0$.

(iii) $\left(H^{*} v_{n, m}\right)(i, j) \geq 0$ for $(i, j) \neq(n, m)$.

Remark. By specifying the entries of the matrices $v_{n, m}$ for each $(n, m)$ we try to fulfill conditions (i), (ii), and (iii). It is surprising that the obvious choice, i.e., when we set $v_{n, m}(i, j)=1$ for each point $(i, j)$ of the triangle $\Delta_{n, m}$, is a failure. Indeed, one may compute that in this case we have

$$
\left(H^{*} v_{n, m}\right)(n, m-1)=-\gamma_{m-1}+\beta_{n}-\beta_{m-1} .
$$

In particular, for $\beta_{i} \equiv 0$, the value $\left(H^{*} v_{n, m}\right)(n, m-1)$ is always negative, i.e., condition (iii) cannot be satisfied.

We need another choice of $v_{n, m}$. By setting $v_{n, m}(i, j)=1$ for every other point in the triangle $\Delta_{n, m}$ and $v_{n, m}(i, j)=0$ for all other points, we obtain Theorem 1 of [9] (see Example 1 of the present paper). On the other hand, if we take $v_{n, m}$ with support on the vertical line in the triangle $\Delta_{n, m}$ we obtain Theorem 1 of [6, p. 423]. Of course, there are plenty of other choices for $v_{n, m}$ (see [6, Theorem 2]). All sufficient conditions known so far required that the sequence $\beta_{n}$ was nondecreasing. In Example 2 we will exhibit a class of polynomials where $\beta_{n}$ is nonincreasing for $n \geq 1$, nonetheless nonnegative linearization holds. This depends on a delicate choice of the matrices $v_{n, m}$.

Proof of Theorem 1. $\quad(\Leftarrow)$ In view of Proposition 1 it suffices to show that the boundary value problem (6) has nonnegative solutions. Assume $u(n, m)$ satisfies (6). We will prove that $u(n, m) \geq 0$ by induction on $m$. Assume $u(i, j) \geq 0$ for $j<m$. Observe that supp $H^{*} v_{n, m} \subset \Delta_{n, m+1}$ and that all the points $(i, j)$ in $\Delta_{n, m+1}$ which are different from $(n, m)$, satisfy $j<m$. Thus

$$
\begin{aligned}
0 & =\left\langle H u, v_{n, m}\right\rangle=\left\langle u, H^{*} v_{n, m}\right\rangle \\
& =\left(H^{*} v_{n, m}\right)(n, m) u(n, m)+\sum_{j<m}\left(H^{*} v_{n, m}\right)(i, j) u(i, j)
\end{aligned}
$$

Hence

$$
-\left(H^{*} v_{n, m}\right)(n, m) u(n, m)=\sum_{j<m}\left(H^{*} v_{n, m}\right)(i, j) u(i, j) \geq 0,
$$

so $u(n, m) \geq 0$.

$(\Rightarrow)$ This part immediately follows from the next lemma.

Lemma. There exists a matrix $v_{n, m}(i, j)$ such that:

(i) $\operatorname{supp} v_{n, m} \subset \Delta_{n, m}$.

(ii) $H^{*} v_{n, m}=\sum_{k=|n-m|}^{\min (n+m, N-1)} g(n, m, k) \delta_{k, 0}-\delta_{n, m}$, where $\delta_{i, j}$ denotes the matrix with entry 1 at $(i, j)$ and zero otherwise. 
Proof of the Lemma. We have

$$
\begin{aligned}
H^{*} \delta_{n, m-1}= & \gamma_{n+1} \delta_{n+1, m-1}+\beta_{n} \delta_{n, m-1}+\alpha_{n-1} \delta_{n-1, m-1} \\
& -\gamma_{m} \delta_{n, m}-\beta_{m-1} \delta_{n, m-1}-\alpha_{m-2} \delta_{n, m-2} .
\end{aligned}
$$

Therefore

$$
\delta_{n, m}=-\frac{1}{\gamma_{m-1}} H^{*} \delta_{n, m-1}+w,
$$

where $w$ is a linear combination of $\delta_{n-1, m-1}, \delta_{n, m-1}, \delta_{n+1, m-1}$, and $\delta_{n, m-2}$. By iterating this formula we obtain that

$$
\delta_{n, m}=-H^{*} v_{n, m}+\sum_{k=|n-m|}^{\min (n+m, N-1)} c_{k} \delta_{k, 0},
$$

where supp $v_{n, m} \subseteq \Delta_{n, m}$. Let $u(n, m)=P_{n}(x) P_{m}(x)$. Then (7) and the fact that $H u=0$ imply

$$
\begin{aligned}
P_{n}(x) P_{m}(x) & =\left\langle u, \delta_{n, m}\right\rangle=-\left\langle u, H^{*} v_{n, m}\right\rangle+\sum_{k=|n-m|}^{\min (n+m, N-1)} c_{k}\left\langle u, \delta_{k, 0}\right\rangle \\
& =\sum_{k=|n-m|}^{\min (n+m, N-1)} c_{k} P_{k}(x) .
\end{aligned}
$$

This implies $c_{k}=g(n, m, k)$.

\section{Examples}

Example 1. Let

$$
v_{n, m}(i, j)= \begin{cases}1, & (i, j) \in \Delta_{n, m},(n+m)-(i+j) \text { odd }, \\ 0, & \text { otherwise. }\end{cases}
$$

The points in the support of $v_{n, m}$ are marked by open circles.

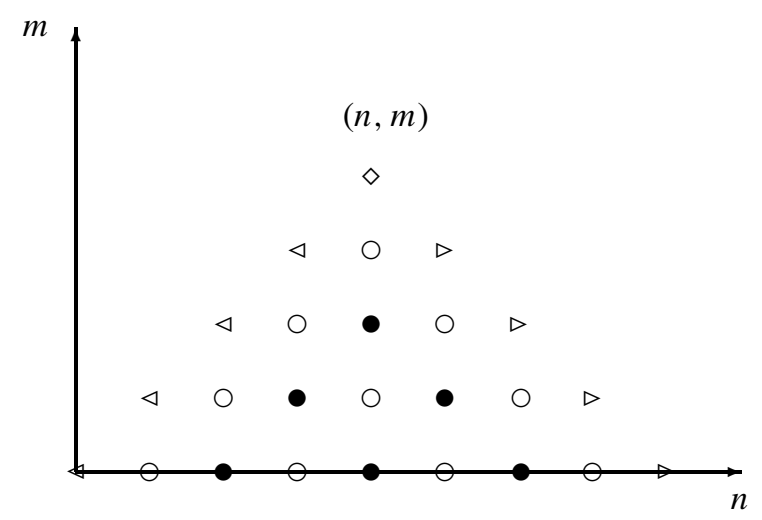


This figure is correct in the case $N=+\infty$ or $n+m \leq N-1$. For $N$ finite and $n+m \geq N$, the corresponding figure is

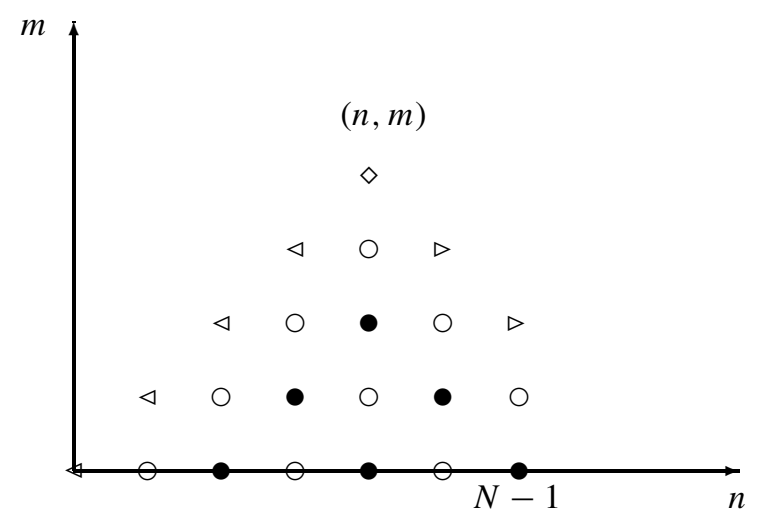

In both cases supp $H^{*} v_{n, m}$ consists of $\circ, \bullet, \triangleleft, \triangleright$, and $\diamond$. A straightforward computation gives

$$
\left(H^{*} v_{n, m}\right)(i, j)= \begin{cases}-\alpha_{m}, & (i, j)=(n, m), \\ \beta_{i}-\beta_{j}, & (i, j)-\diamond, \\ \alpha_{i}+\gamma_{i}-\alpha_{j}-\gamma_{j}, & (i, j)-\bullet, \\ \alpha_{i}-\alpha_{j}, & (i, j)-\triangleright, \\ \gamma_{i}-\alpha_{j}, & (i, j)-\triangleleft .\end{cases}
$$

This implies Theorem 1 of [9].

Example 2. Theorem 1 of [9] and Theorem 1 of [6] require that the sequence $\beta_{n}$ is nondecreasing. Here we will construct examples in which $\beta_{n}$ is decreasing for $n \geq 1$ and the corresponding infinite system of orthogonal polynomials has nonnegative product linearization. These examples are new, and cannot be covered by any of the previously known results. The construction is possible by an appropriate choice of the matrices $v_{n, m}$. The first term of $\beta_{n}$ must satisfy $\beta_{0} \leq \beta_{n}$ because

$$
p_{1} p_{n}=\frac{\gamma_{n}}{\gamma_{0}} p_{n+1}+\frac{\beta_{n}-\beta_{0}}{\gamma_{0}} p_{n}+\frac{\alpha_{n}}{\gamma_{0}} p_{n-1}
$$

Let $\varepsilon_{n}$ be an increasing and concave sequence of negative numbers, i.e.,

$$
\begin{aligned}
\varepsilon_{n} & <0, \\
\varepsilon_{n} & >\varepsilon_{n-1}, \\
\varepsilon_{n+1} & \geq \frac{1}{2} \varepsilon_{n}+\frac{1}{2} \varepsilon_{n+2} .
\end{aligned}
$$


Let $\alpha_{0}=0, \gamma_{0}=1$, and $\alpha_{n}=\gamma_{n}=1$ for $n \geq 1$. Assume that $\beta_{0} \leq 0, \beta_{n}>0$, and $\beta_{n}$ is decreasing for $n \geq 1$. Assume also that

$$
\begin{aligned}
\left(\beta_{0}-\beta_{1}\right) \varepsilon_{0} & \leq 1, \\
\beta_{0} & \leq 2 \varepsilon_{0}-\varepsilon_{1}, \\
\beta_{n} & \leq-\varepsilon_{n}, \\
\beta_{n} & \leq 2 \varepsilon_{n}-\varepsilon_{n-1}-\varepsilon_{n+1},
\end{aligned}
$$

for $n \geq 1$. Define $v_{n, m}$ according to

$$
v_{n, m}(i, j)= \begin{cases}1 & \text { for }(i, j) \in \Delta_{n, m} \text { and }(n+m-i-j) \text { odd, } \\ \varepsilon_{j} & \text { for }(i, j) \in \Delta_{n, m} \text { and }(n+m-i-j) \text { even, } \\ 0 & \text { for }(i, j) \notin \Delta_{n, m} .\end{cases}
$$

It can be checked easily that the matrices $v_{n, m}$ satisfy conditions (i), (ii), and (iii) of Theorem 1 . Therefore the polynomials $p_{n}$ defined by the recurrence relation

$$
x p_{n}=p_{n+1}+\beta_{n} p_{n}+p_{n-1},
$$

where $\beta_{n}$ satisfies (9)-(12), admit nonnegative linearization. Applying this to the sequences $\varepsilon_{n}=-2^{-n-1}$ and $\varepsilon_{n}=-(n+2)^{-1}$ gives that the following two systems admit nonnegative linearization:

$$
\begin{aligned}
x p_{0} & =p_{1}-\frac{3}{4} p_{0}, \\
x p_{n} & =p_{n+1}+\frac{1}{2^{n+2}} p_{n}+p_{n-1}, \\
x q_{0} & =q_{1}-\frac{2}{3} q_{0}, \\
x q_{n} & =q_{n+1}+\frac{1}{(n+2)^{3}} q_{n}+q_{n-1} .
\end{aligned}
$$

Acknowledgment. This work was partially supported by KBN (Poland) under grant 5 P03A 03420.

\section{References}

1. R. ASKEY (1970): Linearization of the product of orthogonal polynomials. In: Problems in Analysis (R. Gunning, ed.). Princeton, NJ: Princeton University Press, pp. 223-228.

2. R. ASKEY (1975): Orthogonal polynomials and special functions. Regional Conference Series in Applied Mathematics, Vol. 21. Philadelphia: SIAM.

3. G. GASPER (1970): Linearization of the product of Jacobi polynomials, I, II. Canad. J. Math., 22:171-175, 582-593.

4. E. HYLLERAAS (1962): Linearization of products of Jacobi polynomials. Math. Scand., 10:189-200.

5. C. MARKETT (1994): Linearization of the product of symmetric orthogonal polynomials. Constr. Approx., 10:317-338.

6. W. MŁOTKOWSKI, R. SZWARC (2001): Nonnegative linearization for polynomials orthogonal with respect to discrete measures. Constr. Approx., 17:413-429.

7. M. RAHMAN (1981): The linearization of the product of continuous q-Jacobi polynomials. Canad. J. Math., 33:961-987. 
8. L. J. ROGERS (1894): Second memoir on the expansion of certain infinite products. Proc. London Math. Soc., 25:318-343.

9. R. SZWARC (1992): Orthogonal polynomials and a discrete boundary value problem, I, II. SIAM J. Math. Anal., 23:959-964, 965-969.

10. R. SzWARC (1996): Nonnegative linearization and quadratic transformation of Askey-Wilson polynomials. Canad. Math. Bull., 39:241-249.

R. Szwarc

Institute of Mathematics

Wrocław University

pl. Grunwaldzki $2 / 4$

50-384 Wrocław

Poland 